\title{
EXPANSION FLOWS ON WALLS WITH NONEQUILIBRIUM CONDENSATION*
}

\author{
BY \\ JOSEPH H. CLARKE (deceased) \\ AND \\ CAN F. DELALE ${ }^{1}$ (Brown University)
}

\begin{abstract}
The streamtube method for supersonic expansion flows on walls with nonequilibrium condensation is developed incorporating the radius dependence on the droplet growth rate. In the presence of an embedded, frozen, oblique shock wave arising from supercritical heat addition from condensation, the method is supplemented by the derived oblique shock relations, and the oblique shock location is determined by employing a shock fitting technique originally introduced by Barschdorff [11]. Some advantages of the proposed streamtube method are explored in comparison with the numerical method of characteristics.
\end{abstract}

I. Introduction. Condensation phenomena have been intensively investigated in the past both experimentally and theoretically in diverse fields like meteorology, supersonic nozzle flow, steam-turbine technology, wind-tunnel applications, cloud chambers, etc. The theory of two-phase condensation phenomena in compressible fluid flow with nonequilibrium thermodynamics was first formulated by Oswatitsch [1]. In present times the subject can be conveniently studied in the recent articles by Wegener [2] and Gyarmathy [3] and in their extensive references.

Until recently almost all of the analytical studies of gas dynamic problems with nonequilibrium condensation have been for one-dimensional steady flow. The first attempt that considers steady two-dimensional supersonic nozzle flow with steam condensation was made by Bartlmä [4], who applied the method of characteristics with a simplified linearized rate equation. Kurshakov [5] et al. studied both experimentally and analytically the water-vapor condensation in a steady two-dimensional PrandtlMeyer flow around a corner using the numerical method of characteristics and the classical homogeneous nucleation and droplet growth theories. Smith [6] investigated the same problem experimentally. Davydov [7] used the same numerical approach in investigating steady two-dimensional flow with condensation. Sislian and Glass [8] also used the numerical method of characteristics in analyzing the shock tube problem with nonequilibrium condensation and with a gas dynamic oblique shock caused by supercritical heat addition from condensation.

*Received March 11, 1987.

${ }^{1}$ Current address: Depaitment of Mathematics, Boğaziçi University, P.K. 2, Bebek, Istanbul, Turkey 
In contrast to the above-mentioned, well-studied numerical methods of calculations based on various theories of condensation, a developed asymptotic method based on the same theories offers analytic structure so that functions over definite, physically distinct regions of space can be compared to measurements mapped over the same regions. According to existing numerical results, the condensation zone is characterized by a lack of coherent, detailed structures. The expected significance of an asymptotic method is that the condensation zone is characterized by distinct, coherent, detailed structures. Recent publications falling in the category of steady quasi-one-dimensional nozzle flow with nonequilibrium condensation that employ the asymptotic approach are by Blythe and Shih [9] and Clarke and Delale [10]. The detailed structure of the flow offered by the asymptotic nature of the solutions in each article is surprisingly promising.

In this investigation the asymptotic analysis for quasi-one-dimensional nozzle flow with nonequilibrium condensation of [10] is extended to solve two-dimensional problems of supersonic expansion flows with nonequilibrium condensation by employing the streamtube formalism. Clearly the flow along the curved arbitrarily small streamtubes in a steady two-dimensional flow can be completely described by the slightly modified equations of quasi-one-dimensional nozzle flow for arbitrarily given area change. The locations and cross-sectional areas of the streamtubes are then determined by introducing a two-dimensional consideration. In cases where the dependent flow variables exhibit discontinuities, as, e.g., across oblique shock waves arising from the compressive heat addition from condensation, the construction of the network of streamtubes is modified by employing the oblique shock relations for condensing flows and using a shock fitting technique originated by Barschdorff [11] and modified analytically by the present authors [12].

Only a formal development of the streamtube method for expansion flows on walls with nonequilibrium condensation is presented. There are no asymptotic analysis and numerical calculations in the present article.

\section{One-dimensional streamtube analysis.}

A. The flow and state equations in natural coordinates. We consider the steady twodimensional supersonic expansion flow of a mixture of a condensible vapor and an inert carrier gas. We focus on a streamtube constructed by two streamlines separated by an arbitrarily small normal distance $\Delta r^{\prime}\left(s^{\prime}\right)$ and of width unity perpendicular to the plane of the streamlines (Fig. 1).

The flow equations together with the thermal and caloric equations of state for two-dimensional steady flow of the mixture in natural coordinates $\left(s^{\prime}, n^{\prime}\right)$ can be written as

continuity: $\quad \rho^{\prime} u^{\prime} \Delta n^{\prime}=\Delta m^{\prime}=$ constant along a streamtube,

$s^{\prime}-$ momentum: $\quad \rho^{\prime} u^{\prime} \frac{\partial u^{\prime}}{\partial s^{\prime}}=-\frac{\partial p^{\prime}}{\partial s^{\prime}}$,

$n^{\prime}-$ momentum: $\quad \rho^{\prime} \frac{u^{\prime^{2}}}{R_{s}^{\prime}}=\rho^{\prime} u^{\prime^{2}} \frac{\partial \theta}{\partial s^{\prime}}=\frac{\partial p^{\prime}}{\partial n^{\prime}}$, 
energy: $\quad h^{\prime}+\frac{1}{2} u^{\prime^{2}}=c_{p 0}^{\prime} T^{\prime}-L^{\prime} g^{\prime}+\frac{1}{2} u^{\prime^{2}}=$ constant along a streamtube,

thermal equation of state: $\quad p^{\prime}=\mathscr{R}\left(\frac{\rho_{i}^{\prime}}{\mu_{i}}+\frac{\rho_{v}^{\prime}}{\mu_{v}}\right) T^{\prime}$

where $\left(u^{\prime}, \theta\right)$ characterizes the magnitude and direction of the flow velocity, $\rho^{\prime}, p^{\prime}$, and $T^{\prime}$ are the mass density, the pressure, and the temperature of the mixture respectively, $\rho_{i}^{\prime}, \rho_{v}^{\prime}$ are the mass densities and $\mu_{i}, \mu_{v}$ are the molecular weights of the inert gas and of the vapor respectively, $\mathscr{R}$ is the universal gas constant, $c_{p 0}^{\prime}$ is the specific heat at constant pressure of the vapor-gas mixture, $L^{\prime}$ is the latent heat of vaporization, $h^{\prime}$ is the tertiary mixture specific enthalpy, $\Delta m^{\prime}$ is the mixture mass flow rate along a streamtube, $g^{\prime}$ is the condensate mass fraction, and $R_{s}^{\prime}$ is the radius of curvature of the streamlines.

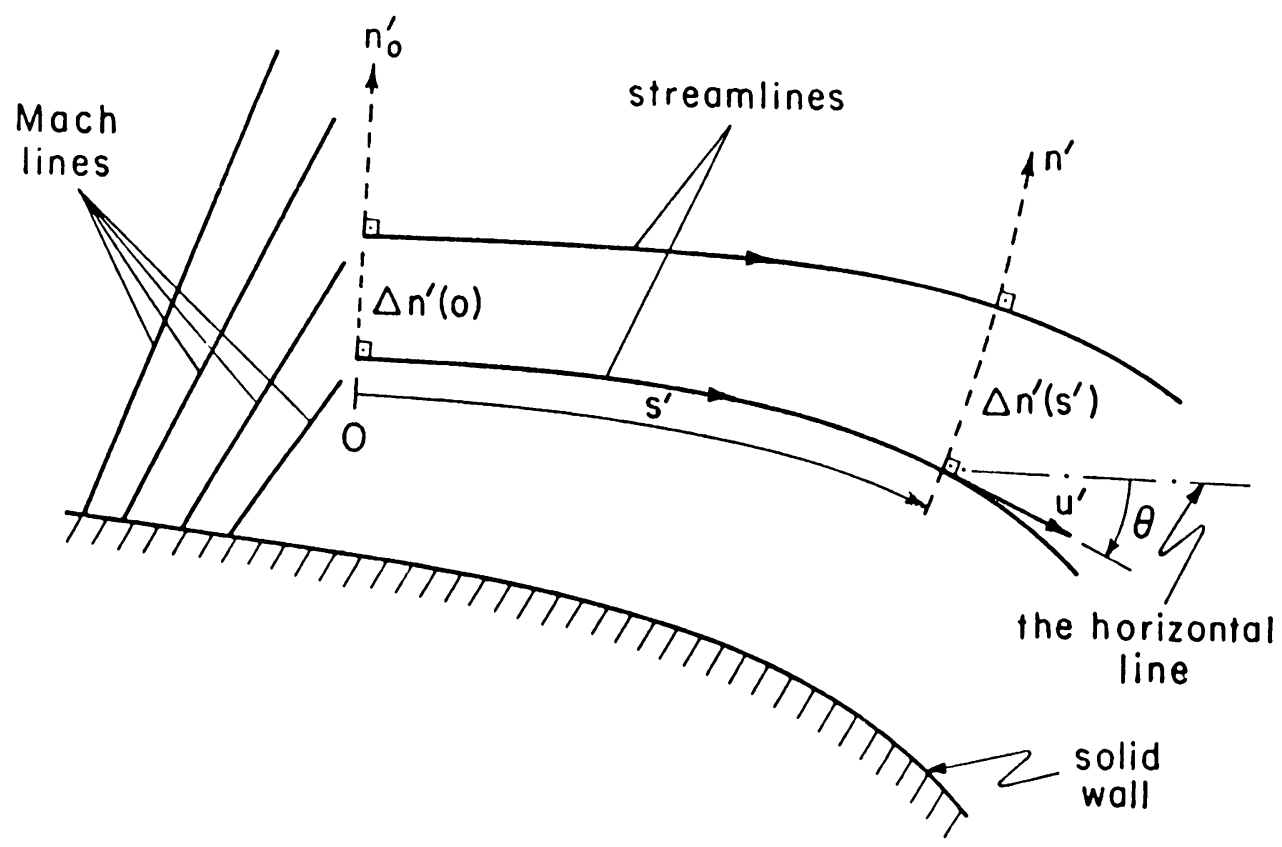

FIG. 1. Natural coordinate frame for a streamtube.

Denoting by subscript $c$ the variables at the saturation point, we carry out a normalization similar to that in quasi-one-dimensional flows, i.e., we define

etc., where

$$
\begin{aligned}
& \rho \equiv \frac{\rho^{\prime}}{\rho_{c}^{\prime}}, \quad p \equiv \frac{p^{\prime}}{p_{c}^{\prime}}, \quad T \equiv \frac{T^{\prime}}{T_{c}^{\prime}}, \quad L \equiv \frac{L^{\prime}}{L_{c}^{\prime}}, \quad g \equiv \frac{\mu_{0} H g^{\prime}}{\mu_{v}}, \\
& c_{p 0} \equiv \frac{\mu_{0}}{\mathscr{R}} c_{p 0}^{\prime} \equiv \frac{\gamma}{\gamma-1}, \quad h \equiv \frac{h^{\prime}}{\left(\mathscr{R} / \mu_{0}\right) T_{c}^{\prime}}, \quad u \equiv \frac{u^{\prime}}{\left(\left(\mathscr{R} / \mu_{0}\right) T_{c}^{\prime}\right)^{1 / 2}}, \\
& n \equiv \frac{n^{\prime}}{s_{0}^{\prime}}, \quad s \equiv \frac{s^{\prime}}{s_{0}^{\prime}}, \quad R_{s} \equiv \frac{R_{s}^{\prime}}{s_{0}^{\prime}}, \\
& \Delta s \equiv \frac{\Delta s^{\prime}}{s_{0}^{\prime}}, \quad \Delta n \equiv \frac{\Delta n^{\prime}}{s_{0}^{\prime}}, \quad A \equiv \frac{\Delta n^{\prime}}{\Delta n_{c}^{\prime}} \equiv \frac{\Delta n}{\Delta n_{c}},
\end{aligned}
$$

and $\Delta m_{v}^{\prime}, \Delta m_{l}^{\prime}$, and $\Delta m^{\prime}$ are the mass flow rates of the vapor, of the dispersed liquid, and of the mixture along a streamtube respectively, $\omega_{0}$ is the initial specific humidity, and $s_{0}^{\prime}$ is a characteristic streamwise flow length. 
With the above normalization, Eqs. (1)-(5) become

$$
\begin{aligned}
& \text { continuity: } \quad \rho u A=u_{c} \text { along a streamtube, } \\
& s \text {-momentum: } \quad \rho u \frac{\partial u}{\partial s}=-\frac{\partial p}{\partial s} \\
& n \text {-momentum: } \quad \frac{\rho u^{2}}{R_{s}}=\frac{\partial p}{\partial n}=\rho u^{2} \frac{\partial \theta}{\partial s} \\
& \text { energy: } c_{p 0} T+\frac{1}{2} u^{2}-L g=c_{p 0}+\frac{1}{2} u_{c}^{2} \text { along a streamtube, } \\
& \text { thermal equation of state: } p=\rho T\left(1-H^{-1} g\right) .
\end{aligned}
$$

Moreover, the continuity equation and the $s$-momentum equation can be combined to yield (for details see [10])

$$
\left(p+\rho u^{2}\right) A=1+u_{c}^{2}+\int_{1}^{A} p d A \quad \text { along a streamtube. }
$$

B. The rate equation along a streamtube. The integral condensate rate equation along a streamtube can be obtained similar to the derivation given in [10]. Let $m_{r}^{\prime}\left(s^{\prime}, \xi^{\prime}\right)$ be the mass of a droplet of radius $r^{\prime}$ at current point $s^{\prime}$ that originated at prior point $\xi^{\prime}$ along a streamtube, and let $J^{\prime}\left(\xi^{\prime}\right)$ be the time rate of production per unit volume at $\xi^{\prime}$ of clusters of critical size along the same streamtube. If the streamtube has an arbitrarily small cross-sectional area $1 \cdot \Delta n^{\prime}\left(s^{\prime}\right)$ at $s^{\prime}$, the integral condensate rate equation can be written as

$$
g^{\prime}\left(s^{\prime}\right) \equiv \frac{\Delta m_{l}^{\prime}\left(s^{\prime}\right)}{\Delta m^{\prime}}=\frac{1}{\Delta m^{\prime}} \int_{-\infty}^{s^{\prime}} d \xi^{\prime} m_{r}^{\prime}\left(s^{\prime}, \xi^{\prime}\right) 1 \cdot \Delta n^{\prime}\left(\xi^{\prime}\right) J^{\prime}\left(\xi^{\prime}\right) \text { along the streamtube, }
$$

where $\Delta m_{l}^{\prime}$ and $\Delta m^{\prime}$ are the dispersed liquid and mixture mass flow rates along the streamtube respectively. From the appropriate functional forms for the nucleation and droplet growth rate equations together with the normalization carried out in detail in the appendix, it follows that the integral condensate rate equation can be conveniently written in normalized form as

$$
g(s)=\lambda^{3} \int_{-\infty}^{s} \mathscr{M}(s, \xi) \Sigma(\xi) A(\xi) \exp \left[-K^{-1} B(\xi)\right] d \xi \quad \text { along a streamtube, }
$$

where

$$
\mathscr{M}(s, \xi) \equiv\left[\int_{\xi}^{s} \Omega(\eta) d \eta\right]^{3 m}, \quad \frac{1}{2} \leq m \leq 1 .
$$

C. Complete set of equations along a streamtube. Equations (7), (10), (11), (12), (14), and (15) form the complete set of equations for the normalized variables $\rho$, $u, p, T$, and $g$ along a streamtube of arbitrarily small cross-sectional area. The $n$-momentum equation, Eq. (9), would be unnecessary if the locations and crosssectional areas of the streamtubes were known; however, the construction of the network of streamtubes and evaluation of the normalized cross-sectional area $A(s)$ along each streamtube require simultaneous consideration of the $n$-momentum equation. These are treated in the next section. 
Equations (7), (10), (11), (12), (14), and (15) along a streamtube are identical with the governing equations of quasi-one-dimensional nozzle flows with nonequilibrium condensation of [10] if the streamwise coordinate $s$ is replaced by the nozzle axial coordinate $x$ and if the normalized area $A(s)$ is thought to act as the given nozzle cross-sectional area $A(x)$. (In [10] the solution is given for $m=1$.) The formalism and solution for quasi-one-dimensional nozzle flows with nonequilibrium condensation then apply along each streamtube given the cross-sectional area $A(s)$. The simultaneous evaluation of $A(s)$ and the construction of the network of streamtubes for steady supersonic expansion flows over curved solid boundary surfaces are discussed below.

\section{Construction of the network of streamtubes for smooth flows.}

A. Area-direction relation. We consider two adjacent streamtubes of normalized cross-sectional areas $\Delta n(s)$ and $\Delta n_{1}(s)$ (Fig. 2). We use the natural coordinate frame along each streamtube and seek a geometric relation between the cross-sectional area and the flow direction.

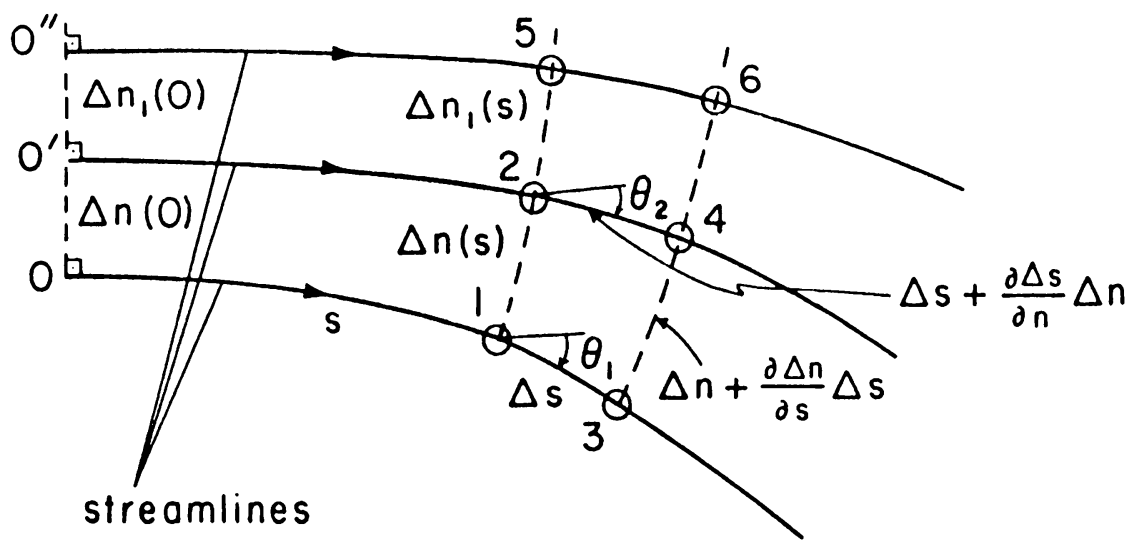

Fig. 2. Construction of the network of streamtubes in natural coordinates.

From the geometry of Fig. 2, it follows that

$$
\theta_{1}-\theta_{2}=\partial \Delta n / \partial s
$$

where

$$
\theta_{1} \equiv \theta(s, n) \quad \text { and } \quad \theta_{2} \equiv \theta(s, n+\Delta n)=\theta(s, n)+(\partial \theta / \partial n) \Delta n
$$

correct to first order in $\Delta n$. Thus we may choose to write Eq. (16) in the conventional form

$$
\frac{1}{\Delta n} \frac{\partial \Delta n}{\partial s}=-\frac{\partial \theta}{\partial n}
$$

Furthermore if we consider the relation $A(s) \equiv \Delta n(s) / \Delta n_{c}$, we obtain the areadirection relation by direct substitution into Eq. (17):

$$
\frac{1}{A} \frac{\partial A}{\partial s}=-\frac{\partial \theta}{\partial n}
$$


B. Locations of streamtubes and method of solution. The construction of the network of streamtubes will logically start on the prescribed curved solid boundary surface along which the flow expands. The curved surface can be taken as the lower streamline for the construction of the first streamtube. The construction of the first streamtube is completed if the upper streamline of the two-dimensional flow field can be drawn for an arbitrarily small initial normal separation. The upper streamline of the constructed streamtube then acts as the given lower streamline for the next streamtube to be constructed. Thus the network of streamtubes can be generated successively.

Hence our objective lies in constructing the upper streamline given the lower streamline in the plane of the two-dimensional flow field of a streamtube with an arbitrarily small initial cross-sectional area. For this reason we consider an initial supersonic flow field designated by points $0,0^{\prime}, 0^{\prime \prime}$, etc., and separated by arbitrarily small normal distances $\Delta n(0), \Delta n_{1}(0)$, etc. We assume that the lower streamline is known. (For initial calculations the lower streamline can be taken as the prescribed curved solid boundary surface.) For constructing the upper streamline passing through $0^{\prime}$, we first consider the rate of change in the cross-sectional area of the streamtube under construction as we advance downstream along the lower streamline. We suppose that the normalized cross-sectional area $\Delta n(s)$ or $A(s)$ of the streamtube under construction at point 1 on the lower streamline with coordinates $(s, n)$ is known. We ask for the variation in the cross-sectional area as we advance an arbitrarily small normalized distance $\Delta s$ along the lower streamline to arrive at point 3 with coordinates $(s+\Delta s, n)$ (see Fig. 2). It follows from the area-direction relation, Eq. (18), that

$$
A(s+\Delta s)=A(s)[1-(\partial \theta / \partial n) \Delta s] .
$$

The derivative $\partial \theta / \partial n$ at $(s, n)$ can be approximated by the forward difference formula

$$
\frac{\partial \theta}{\partial n} \simeq \frac{\theta(s, n+\Delta n)-\theta(s, n)}{\Delta n(s)}=\frac{\left(\theta_{2}-\theta_{1}\right)}{\Delta n_{c} A(s)} .
$$

By substitution from Eq. (20) into Eq. (19), we find that the normalized area at $(s+\Delta s, n)$, i.e., at point 3 , is related to the normalized area at $(s, n)$, i.e., at point 1 , by

$$
A(s+\Delta s)=A(s)+\left(\theta_{1}-\theta_{2}\right) \Delta s / \Delta n_{c} .
$$

We now suppose that upstream of the point $(s, n)$, point 1 in Fig. 2, the network of streamtubes and the flow field are known (e.g., $s=0$ corresponds to the given initial flow field where the network starts). Let us try to extend the construction of the network of streamtubes as we advance an arbitrarily small distance $\Delta s$ along the given (or already constructed) lower streamline to arrive at point 3 in Fig. 2. For this reason we first draw the normal line of length $\Delta n_{c} A(s+\Delta s)$ given by Eq. (21) from point 3 to arrive at point 4 (see Fig. 2). To be able to extend the construction of the upper streamline from point 2 to point 4 , we need to know the direction $\theta_{4}$ of the flow velocity at point 4 . This can only be achieved by introducing the second dynamical consideration needed for two-dimensional flows, i.e., the $n$-momentum 
equation. Let us consider the $n$-momentum equation at point 2 :

$$
\rho_{2} u_{2}^{2}\left(\frac{\partial \theta}{\partial s}\right)_{2}=\left(\frac{\partial p}{\partial n}\right)_{2} .
$$

We may now evaluate $(\partial \theta / \partial s)_{2}$ by a forward difference formula as

$$
\left(\frac{\partial \theta}{\partial s}\right)_{2} \simeq \frac{\left(\theta_{4}-\theta_{2}\right)}{\Delta s+(\partial \Delta s / \partial n) \Delta n} \simeq \frac{\left(\theta_{4}-\theta_{2}\right)}{\Delta s}
$$

where the denominator is simplified since $(\partial \Delta s / \partial n) \Delta n$ is of higher order compared to $\Delta s$. Now $(\partial p / \partial n)_{2}$ has to be approximated by a central difference formula since a forward difference formula would ignore the influence of the pressure distribution (hence of the flow field) of the lower streamline. Thus we have

$$
\left(\frac{\partial p}{\partial n}\right)_{2} \simeq \frac{p_{5}-p_{1}}{\Delta n(s)+\Delta n_{1}(s)}
$$

referring to Fig. 2. Substituting from Eqs. (23) and (24) into Eq. (22) and solving for $\theta_{4}$ yield

$$
\theta_{4} \simeq \theta_{2}+\frac{\left(p_{5}-p_{1}\right)}{\rho_{2} u_{2}^{2}} \cdot \frac{\Delta s}{\Delta n(s)+\Delta n_{1}(s)}
$$

In Eq. (25) all the variables at $s$ and arbitrary $n$, i.e., $\theta_{1}, \theta_{2}, \rho_{1}, \rho_{2}, u_{1}, u_{2}, p_{1}, p_{2}, p_{5}$, $\Delta n(s), \Delta n_{1}(s)$, etc. are known, $\Delta s$ is chosen as small as is necessary in the calculations, and $\theta_{3}$ is known since the lower streamline is given. Thus $\theta_{4}$ can be easily calculated from Eq. (25). The locus of the directions characterized by $\theta_{2}$ and $\theta_{4}$ extend the upper streamline from point 2 to point 4 , hence extending the construction of the first streamtube from $s$ (point 1) to $s+\Delta s$ (point 3) along the given lower streamline. The extension of the network of the next streamtubes from $s$ (points 2,5 , etc.) to $s+\Delta s$ (points 4,6 , etc.) follows in the same manner. The flow variables at $s+\Delta s$ (points $3,4,6$, etc.) can then be evaluated by substituting from the area-direction relation of each streamtube into the quasi-one-dimensional flow equations of Sec. II.C and by following the procedure given in [10] along each streamtube.

In this way, starting with the initial supersonic flow field at $s=0$ and moving downstream along the prescribed curved solid boundary surface, we can generate the network of streamtubes, and by applying the quasi-one-dimensional solution of [10] along each constructed streamtube, we can simultaneously obtain the solution for the flow field.

C. Convergence and stability. In the preceding paragraphs we have outlined the streamtube method for solving two-dimensional supersonic expansion flows with nonequilibrium condensation by employing the one-dimensional asymptotic solution of [10] along each streamtube and by evaluating the local area change by a finite difference scheme from the area-direction relation, Eq. (18), and the $n$-momentum equation, Eq. (22). The error encountered in the flow field along each streamtube arising from the asymptotic nature of the solution for one-dimensional flows has already been discussed in [10]. Therefore we herein discuss only the error resulting from the evaluation of the local area change and of the flow direction by the finite difference scheme. It is well-known $[13,14]$ that this error consists of the discretization error and the stability error. 
For smooth flows $\partial p / \partial n$ and $\partial \theta / \partial n$ are of $O(1)$ numerically, and it follows from Eqs. (20) and (24) that for reasonable normal separations of the streamlines we should have

$$
\theta_{1}-\theta_{2}=O(\Delta n)=O\left(\Delta n_{c}\right) \text { and } p_{5}-p_{1}=O\left(\Delta n+\Delta n_{1}\right)
$$

numerically. By virtue of Eqs. (20)-(25) this suggests that

$$
A(s+\Delta s)=A(s)+O(\Delta s), \quad \theta_{4}=\theta_{2}+O(\Delta s)
$$

numerically. As $\Delta n \rightarrow 0$ and $\Delta s \rightarrow 0$, it follows from Eqs. (26) and (27) that $\theta_{1} \rightarrow \theta_{2}$, $\theta_{4} \rightarrow \theta_{2}$, and $A(s+\Delta s) \rightarrow A(s)$. Thus we have local convergence. It is now obvious that the discretization error depends on the relative local sizes of $\Delta n, \Delta n_{1}$, and $\Delta s$. The smaller they are, the smaller is the discretization error. In particular, $\Delta s$ should be of $o\left(K^{1 / 2}\right)$ on measure $K$ in the rapid growth, onset, and nucleation zones and of $o\left(K^{1 / 3}\right)$ on measure $K$ in the droplet growth zone along each streamtube referring to Eq. (27) above and to Eqs. (77) and (130f) of [10] for a reasonably acceptable solution.

The stability error, on the other hand, actually depends on the cumulative roundoff errors encountered in evaluating local area changes and directions of the flow field. In the method of solution proposed, the round-off errors encountered will have only local effects since they are smoothed out locally by the asymptotic solution of the one-dimensional equations (7)-(12) presented in [10]; however, $\Delta s$ and $\Delta n$ still need to be chosen in such a way that the local round-off errors remain less in magnitude than the local asymptotic error of [10] for a stable solution.

IV. Construction of the network of streamtubes with an embedded oblique shock wave. The two-dimensional steady supersonic expansion of a mixture of a condensible vapor and an inert carrier gas discussed in detail in the preceding section using the streamtube method was developed on the assumption that all the dependent flow variables were continuous throughout the flow field. In cases where the dependent variables of the flow field exhibit discontinuities, as, e.g., across shock waves, the formalism and method of solution of Sec. III are no longer applicable and should be supplemented by the appropriate jump conditions.

In the supersonic expansion of a mixture of a condensible vapor and an inert carrier gas, embedded, frozen, gas dynamic shock waves appear due to the compressive heat addition from condensation for relatively high initial specific humidity or due to downstream boundary conditions. Shock waves in flows caused by compressive heat addition have been recently investigated by Sislian and Glass [8] in the rarefaction region of the shock tube problem employing Lax's [15] smoothing method for shock fitting and by Barschdorff [11] for one-dimensional nozzle flows using an iterative shock fitting technique consistent with the second law of thermodynamics. More recently the present authors [12] have extended Barschdorff's one-dimensional iterative shock fitting technique into a noniterative analytical framework for nozzle flows.

In this section we develop the theory of frozen, gas dynamic, oblique shock waves in condensing flows in detail. We show how the streamtube method of Sec. III can be supplemented for the construction of the network with an embedded, frozen, gas 


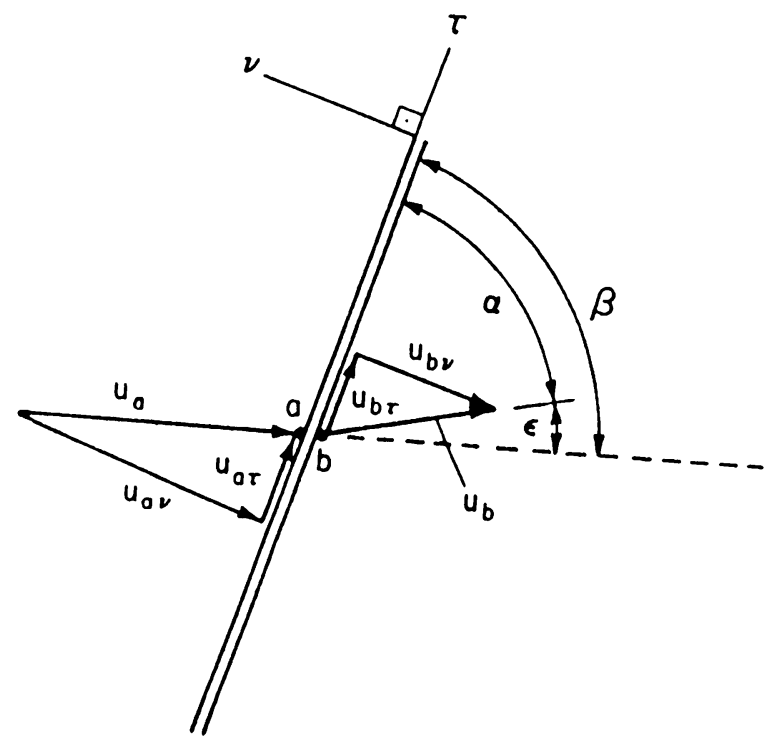

FIG. 3. Geometry of an oblique shockwave.

dynamic, oblique shock wave located by a shock fitting technique first introduced by Barschdorff [11].

A. Analysis of frozen oblique shock waves in condensing flows. We consider the supersonic expansion of a mixture of a condensible vapor and an inert gas at a certain Mach number $M$, based on a typical speed of sound $\sqrt{\gamma T}$. We assume that in the droplet growth zones of streamtubes supercritical flow conditions are reached such that the flow exceeds the thermally choked condition for continuous flow and such that an oblique shock wave appears due to the heat addition from condensation. We let subscripts $a$ and $b$ denote, respectively, quantities upstream and downstream of the shock wave at the shock front (Fig. 3). We also let subscript $\nu$ denote the component normal to the shock wave and subscript $\tau$ denote the component tangent to the shock wave. If $\beta$ is the angle between $u_{a}$ and the shock wave and $\alpha$ is the angle between $u_{b}$ and the shock wave as shown in Fig. 3, $\alpha$ will be different from $\beta$ and a deviation in the flow direction defined by $\varepsilon \equiv \beta-\alpha$ will occur. Projecting the velocities $u_{a}$ and $u_{b}$ in the direction $\nu$ normal to the shock surface and $\tau$ tangent to the shock surface and indicating by subscripts $\nu$ and $\tau$ the corresponding components, we have

$$
u_{a \nu}=u_{a} \sin \beta, \quad u_{a \tau}=u_{a} \cos \beta
$$

and

$$
u_{b \nu}=u_{b} \sin \alpha, \quad u_{b \tau}=u_{b} \cos \alpha
$$


The conservation of mass, momentum, and energy together with the thermal equation of state across the shock surface yield

$$
\begin{aligned}
& \text { continuity: } \rho_{a} u_{a \nu}=\rho_{b} u_{b \nu}, \\
& \nu \text { - momentum: } \quad p_{a}+\rho_{a} u_{a \nu}^{2}=p_{b}+\rho_{b} u_{b \nu}^{2}, \\
& \tau \text { - momentum: } u_{a \tau}=u_{b \tau}, \\
& \text { energy: } \quad \frac{\gamma}{(\gamma-1)} T_{a}+\frac{1}{2} u_{a}^{2}-L_{a} g^{*}=\frac{\gamma}{(\gamma-1)} T_{b}+\frac{1}{2} u_{b}^{2}-L_{b} g^{*}, \\
& \text { thermal equation of state: } \quad \frac{p_{a}}{\rho_{a} T_{a}}=\frac{p_{b}}{\rho_{b} T_{b}}=1-H^{-1} g^{*} .
\end{aligned}
$$

In deriving the above conservation laws together with the thermal equation of state, the flow is assumed to be frozen through the shock wave at $g=g^{*}$. Eliminating the temperature between Eqs. (33) and (34) and utilizing Eq. (32), Eqs. (33) and (34) can be combined into an equation of the form

$$
\frac{\gamma p_{a}}{(\gamma-1) \rho_{a}\left(1-H^{-1} g^{*}\right)}+\frac{1}{2} u_{a}^{2}=\frac{\gamma p_{b}}{(\gamma-1) \rho_{b}\left(1-H^{-1} g^{*}\right)}+\frac{1}{2} u_{b}^{2}+\Delta L g^{*},
$$

where we have defined $\Delta L$ by

$$
\Delta L \equiv L_{a}-L_{b}
$$

We also note that

$$
M_{a}^{2} \equiv \frac{u_{a}^{2}}{\gamma T_{a}}=\frac{\rho_{a} u_{a}^{2}\left(1-H^{-1} g^{*}\right)}{\gamma p_{a}}
$$

and

$$
M_{b}^{2} \equiv \frac{u_{b}^{2}}{\gamma T_{b}}=\frac{\rho_{b} u_{b}^{2}\left(1-H^{-1} g^{*}\right)}{\gamma p_{b}} .
$$

The solution of the above oblique shock relations as worked out in detail in [16] leads to the following relations:

$$
\begin{aligned}
M_{b \nu}^{2}= & {\left[\frac{F\left(M_{a \nu}^{2}\right)}{2(\gamma-1)}-\gamma\left(1-H^{-1} g^{*}\right)\right.} \\
& \left. \pm\left\{\left[\frac{F\left(M_{a \nu}^{2}\right)}{2(\gamma-1)}\right]^{2}-\left(1-H^{-1} g^{*}\right)\left[\frac{\gamma}{(\gamma-1)}-\frac{\left(1-H^{-1} g^{*}\right)}{2}\right] F\left(M_{a \nu}^{2}\right)\right\}^{1 / 2}\right] \\
& \times\left[\gamma^{2}-\frac{F\left(M_{a \nu}^{2}\right)}{2}\right]^{-1},
\end{aligned}
$$

where

$$
\begin{gathered}
F\left(M_{a \nu}^{2}\right)=\frac{\left(1-H^{-1} g^{*}+\gamma M_{a \nu}^{2}\right)^{2}}{\left(1 /(\gamma-1)-\Delta L g^{*} / \gamma T_{a}\right) M_{a \nu}^{2}+(1 / 2) M_{a \nu}^{4}}, \\
M_{a \nu}^{2}=M_{a}^{2} \sin ^{2} \beta, \\
M_{b \nu}^{2}=M_{b}^{2} \sin ^{2} \alpha,
\end{gathered}
$$


and

$$
\begin{aligned}
\frac{\rho_{b}}{\rho_{a}} & =\frac{\left(1-H^{-1} g^{*}+\gamma M_{b \nu}^{2}\right) M_{a \nu}^{2}}{\left(1-H^{-1} g^{*}+\gamma M_{a \nu}^{2}\right) M_{b \nu}^{2}}, \\
\frac{p_{a}}{p_{b}} & =\frac{\left(1-H^{-1} g^{*}+\gamma M_{b \nu}^{2}\right)}{\left(1-H^{-1} g^{*}+\gamma M_{a \nu}^{2}\right)}, \\
\frac{T_{a}}{T_{b}} & =\frac{M_{a \nu}^{2}\left(1-H^{-1} g^{*}+\gamma M_{b \nu}^{2}\right)^{2}}{M_{b \nu}^{2}\left(1-H^{-1} g^{*}+\gamma M_{a \nu}^{2}\right)^{2}} \\
\alpha & =\tan ^{-1}\left\{\frac{M_{b \nu}^{2}\left(1-H^{-1} g^{*}+\gamma M_{a \nu}^{2}\right)}{M_{a \nu}^{2}\left(1-H^{-1} g^{*}+\gamma M_{b \nu}^{2}\right)} \tan \beta\right\}, \\
\varepsilon & =\beta-\alpha .
\end{aligned}
$$

The Mach number $M$ that appears in the above expressions is based on a typical speed of sound defined by $\sqrt{\gamma T}$ and is related to the Mach number, based on the local frozen speed of sound with condensation and herein designated by $M_{g}$, by the expression

$$
M_{g}^{2} \equiv \frac{u^{2}}{a_{f}^{2}}=M^{2} \frac{\left(c_{p 0}+H^{-1} g-1\right)}{\left(c_{p 0}-1\right)\left(1-H^{-1} g\right)} .
$$

Equations (39)-(47) permit implicit calculation of all physical properties of the flow downstream of the shock as functions of similar properties upstream of the shock at the shock front if one condition of shock and the frozen condensate mass fraction $g^{*}$ are known. Usually the one condition of shock can be taken as the inclination of the oblique shock wave or the deviation in the flow direction. On the other hand, the solution given above is implicit since $L$ in general depends on the temperature. The dependence of the latent heat on temperature may have important implications on the nature and structure of the shock wave.

When $\Delta L \equiv L\left(T_{a}\right)-L\left(T_{b}\right)=0$ (no jump in the latent heat of vaporization), the solution given by Eq. (39) with one of the signs chosen will correspond to the continuous or no shock solution and with the other sign chosen will correspond to the solution of a frozen, gas dynamic, oblique shock wave across which the latent heat of vaporization does not change.

When $\Delta L \neq 0$, both signs in Eq. (39) yield discontinuities in the flow variables and correspond in reality to shock waves structured by diffusion due to temperature gradients with internal heat exchange from property change. The solution of both signs of Eq. (39) in this case should be obtained iteratively. We may first guess a value for $\Delta L$ and then check if the shock relations are compatible with this value of $\Delta L$. The procedure should be continued until compatibility with a certain degree of accuracy is reached. In cases where $\Delta L$ is relatively small, the strength of the shock wave corresponding to one of the signs in Eq. (39) will be very weak (almost continuous solution), and the discontinuous solution with the other sign chosen will approach the discontinuous solution with $\Delta L=0$.

B. General features of streamtube analysis with shocks in condensing flows. The streamtube formalism and method of solution developed above in Sec. III for supersonic expansion flows with nonequilibrium condensation apply when the mixture 
of the condensible vapor and the inert carrier gas has relatively low initial specific humidity. For sufficiently high initial specific humidity (say, for fixed initial relative humidity and temperature) compressive flow conditions may be reached in the droplet growth zones of streamtubes under construction such that an embedded, frozen, oblique shock wave due to the release of heat of condensation occurs. In this case the inclusion of such a shock wave in the droplet growth zones of the streamtubes becomes necessary to redirect a flow sufficiently diverted by large enough heat addition.

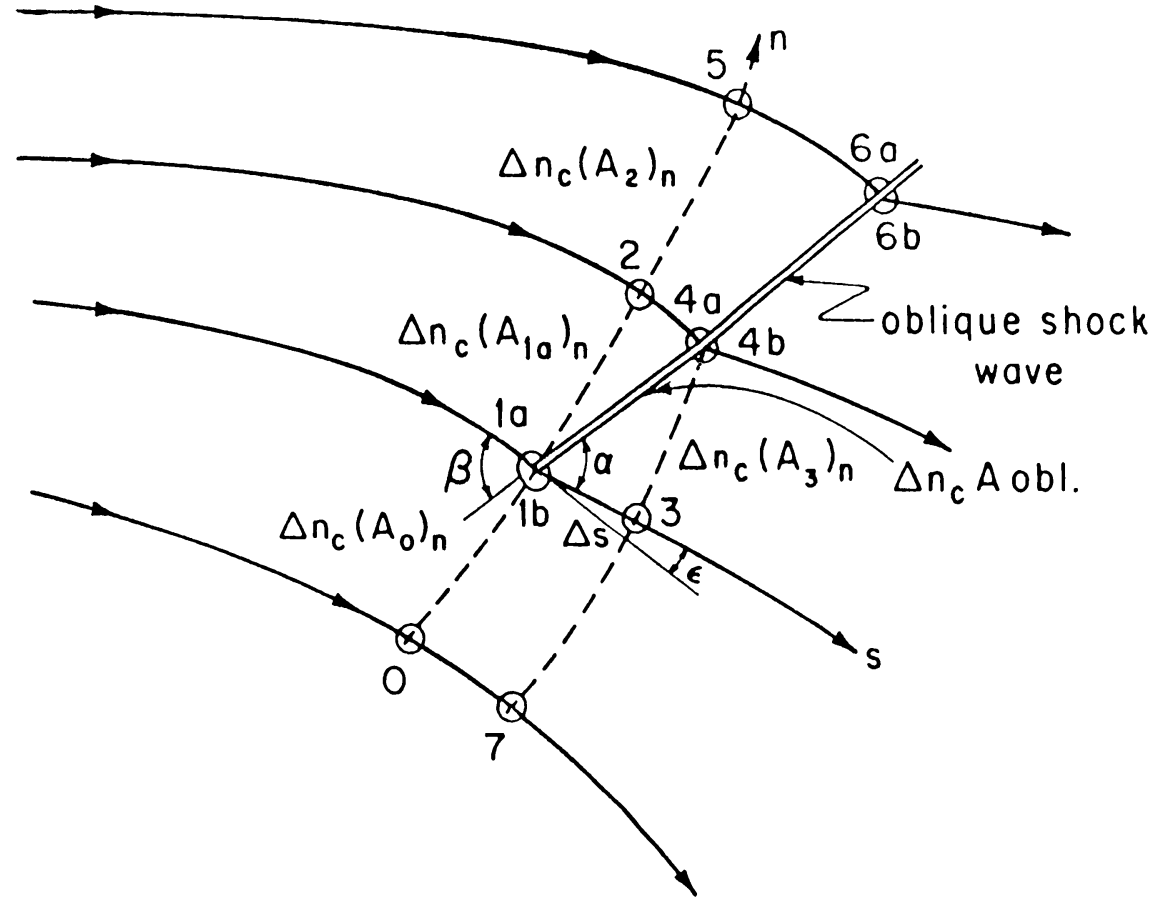

Fig. 4. Configuration of streamtubes with an embedded, frozen, gas dynamic, oblique shockwave.

Wo let point 1 denote the initial formation of an oblique shock wave by compressive condensation in the droplet growth zone of a streamtube (Fig. 4). The formalism and method of solution developed above then apply upstream of point 1 . To be able to continue the construction of the streamtubes downstream of point 1 , we need to supplement the formalism of Sec. III with the equations of oblique shock waves. From the equations of oblique shock waves discussed in detail in Part A of this section, the flow properties downstream of the shock wave at $1 \mathrm{~b}$ can be obtained in terms of similar flow properties upstream of the shock wave at la provided that the angle of inclination $\beta$ and the frozen condensate mass fraction $g^{*}$ at point 1 are known. The equation for $\beta$ should be determined so as to satisfy the conservation of mass along each streamtube, and the value of $g^{*}$ should be determined by shock fitting. The construction of the network of streamtubes downstream of the shock wave requires separate consideration. 
C. Equation for $\beta$. The oblique shock wave appears to redirect the flow diverted by rapid heat addition so as to conserve mass along each streamtube. Thus the inclination of the shock wave is determined naturally by satisfying the continuity equation for streamtubes at the shock location. Across the shock the normalized oblique area, herein denoted by $A_{\text {obl. }}$, is continuous, but the normal area which appears in the continuity equation is discontinuous. We assume that the shock wave diagram is a piecewise linear curve, a time-honored assumption for numerical work, as shown in Fig. 4.

Using the notation and shock structure of the droplet growth zone of [12] and denoting the flow properties upstream and downstream of the shock at the shock front by subscripts $a$ and $b$ respectively, it follows from the continuity equation at point 1 , where the shock is initially formed, that

$$
\left(A_{1 b}\right)_{n}=\frac{\bar{\rho}_{1 a}}{\bar{\rho}_{1 b}} \frac{\bar{u}_{1 a}}{\bar{u}_{1 b}}\left(A_{1 a}\right)_{n} .
$$

In Eq. (49), $\left(A_{1 b}\right)_{n}$ is the normalized area at the shock location of the streamtube continued analytically from downstream as if there were no shocks. Moreover it is evident from the geometry of Fig. 4 that

$$
\left(A_{3}\right)_{n}=A_{\text {obl. }} \sin \alpha
$$

and

$$
\left(A_{1 a}\right)_{n}=A_{\text {obl. }} \sin \beta .
$$

Eliminating the oblique area $A_{\text {obl. }}$ between Eqs. (50) and (51), we have

$$
\left(A_{3}\right)_{n}=\frac{\sin \alpha}{\sin \beta}\left(A_{1 a}\right)_{n} .
$$

It also follows from the area-direction relation of Sec. III, Eq. (19), that

$$
\left(A_{3}\right)_{n}=\left(A_{1 b}\right)_{n}\left[1-(\partial \theta / \partial n)_{1 b} \Delta s\right]
$$

where, as shown in Fig. $4, \Delta s$ is given by

$$
\Delta s=\Delta n_{c} A_{\text {obl. }} \cos \alpha=\Delta n_{c}\left(A_{1 a}\right)_{n} \frac{\cos \alpha}{\sin \beta} .
$$

To evaluate $(\partial \theta / \partial n)_{1 b}$, we must use a backward finite difference formula since a forward finite difference formula for normal derivatives would mean crossing the shock, which is not allowed. Thus we can write

$$
\left(\frac{\partial \theta}{\partial n}\right)_{1 b}=\frac{\theta_{1 b}-\theta_{0}}{\Delta n_{c}\left(A_{0}\right)_{n}}=\frac{\theta_{1 a}-\varepsilon-\theta_{0}}{\Delta n_{c}\left(A_{0}\right)_{n}}
$$

where $\theta_{0}$ and $\left(A_{0}\right)_{n}$ are known from the previous construction of the network of streamtubes and $\theta_{1 b}$ is replaced by $\theta_{1 a}-\varepsilon$. Substitution from Eqs. (54) and (55) into Eq. (53) yields the geometric relation

$$
\left(A_{3}\right)_{n}=\left(A_{1 b}\right)_{n}\left[1-\left(\theta_{1 a}-\varepsilon-\theta_{0}\right) \frac{\left(A_{1 a}\right)_{n}}{\left(A_{0}\right)_{n}} \frac{\cos \alpha}{\sin \beta}\right] .
$$


If we now substitute from Eq. (49) for $\left(A_{1 b}\right)_{n}$ and from Eq. (52) for $\left(A_{3}\right)_{n}$ into Eq. (56), we obtain

$$
\frac{\sin \alpha}{\sin \beta}=\frac{\bar{\rho}_{1 a} \bar{u}_{1 a}}{\bar{\rho}_{1 b} \bar{u}_{1 b}}\left[1-\left(\theta_{1 a}-\varepsilon-\theta_{0}\right) \frac{\left(A_{1 a}\right)_{n} \cos \alpha}{\left(A_{0}\right)_{n} \sin \beta}\right] .
$$

We also recall from the solution of the oblique shock relations discussed in Part A of this section that

$$
\begin{aligned}
\alpha & =\alpha\left(\beta, g^{*}, \text { flow properties at } 1 \mathrm{a}\right), \\
\varepsilon & =\varepsilon\left(\beta, g^{*}, \text { flow properties at } 1 \mathrm{a}\right), \\
\bar{\rho}_{1 a} & =\mathscr{F}_{1}\left(\beta, g^{*}, \text { flow properties at } 1 \mathrm{a}\right), \\
\frac{\bar{\rho}_{1 b}}{\bar{u}_{1 a}} & =\mathscr{F}_{2}\left(\beta, g^{*}, \text { flow properties at } 1 \mathrm{a}\right) . \\
\overline{\bar{u}}_{1 b} &
\end{aligned}
$$

In addition $\theta_{0},\left(A_{0}\right)_{n}$, and $\left(A_{1 a}\right)_{n}$ are known from the previous construction of streamtubes up to the shock front. Therefore, Eq. (57) together with Eqs. (58)-(61) relates $\beta$ to $g^{*}$ in terms of the flow properties at $1 \mathrm{a}$ and can be written as

$$
\beta=\beta\left[g^{*}, \theta_{0},\left(A_{0}\right)_{n}, \text { flow properties at } 1 \mathrm{a}\right] .
$$

Equation (62) completes the determination of the flow properties downstream of the shock as functions of similar properties upstream of the shock provided that $g^{*}$ is known. The procedure can be repeated along each streamtube that crosses the shock in precisely the same manner.

D. Construction of the network of streamtubes downstream of the shock. The construction of the network of streamtubes downstream of the shock would naturally follow the procedure and method of solution discussed in detail in Sec. III. The solution of the oblique shock relations given in Part A of this section together with Eq. (62) for $\beta$ determine the flow properties downstream of the shock for a given value of $g^{*}$ along each streamtube. Very rapid droplet growth continues downstream of the shock; therefore, the droplet growth shock structure solution downstream of the oblique shock is essential for the downstream construction of the network of streamtubes. Although the continuous droplet growth shock structure solution discussed in [10] is no longer valid downstream of the shock because of the discontinuous flow properties across the shock, the droplet growth shock structure relations will still hold downstream of the shock if the normalized area is expanded as

$$
A(s)=\left(A_{1 b}\right)_{n}\left\{1+\left(\frac{1}{A} \frac{\partial A}{\partial s}\right)_{1 b}\left(s-s^{*}\right)+\cdots\right\},
$$

where $\left(A_{1 b}\right)_{n}$ is given by Eq. (49) and $s^{*}$ characterizes the location of the shock and is related to the onset length $s_{k}$ along the streamtube under construction by

$$
s^{*}=s_{k}+l^{-1} K^{1 / 3} \int_{0}^{\left(g^{*}\right)^{1 / 3}} \frac{d \sigma}{\Omega^{*}(\sigma ; K)} .
$$

The definitions of $l$ and $\Omega^{*}$ are precisely those given in [10] and include only properties upstream of the shock. Referring to [12], the droplet growth shock structure 
flow relations can be conveniently written as

$$
\begin{gathered}
\bar{\rho}(\chi) \bar{u}(\chi)=\bar{\rho}_{1 b} \bar{u}_{1 b}, \\
\bar{p}(\chi)+\bar{\rho}(\chi) \bar{u}^{2}(\chi)=\bar{p}_{1 b}+\bar{\rho}_{1 b} \bar{u}_{1 b}^{2}, \\
c_{p 0} \frac{\bar{p}(\chi)}{\bar{\rho}(\chi)\left(1-H^{-1} \bar{g}(\chi)\right)}+\frac{1}{2} \bar{u}^{2}(\chi)-\bar{L}(\chi) \bar{g}(\chi) \\
=c_{p 0} \frac{\bar{p}_{1 b}}{\bar{\rho}_{1 b}\left(1-H^{-1} g^{*}\right)}+\frac{1}{2} \bar{u}_{1 b}^{2}-\bar{L}_{1 b} g^{*}
\end{gathered}
$$

along the streamtube under consideration, where $\chi$ is defined by

$$
\chi \equiv l K^{-1 / 3}\left(s-s^{*}\right)
$$

As worked out in detail in [16], the solution of Eqs. (65)-(67) yields

$$
\bar{u}\left(\bar{g}, g^{*}\right)=\frac{c_{p 0}\left(\bar{p}_{1 b}+\bar{\rho}_{1 b} \bar{u}_{1 b}^{2}\right)-\sqrt{\Delta\left(\bar{g}, g^{*}\right)}}{2 \bar{\rho}_{1 b} \bar{u}_{1 b}\left\{c_{p 0}-\frac{1}{2}\left(1-H^{-1} \bar{g}\right)\right\}}
$$

where

$$
\begin{aligned}
\Delta\left(\bar{g}, g^{*}\right)= & c_{p 0}^{2}\left(\bar{p}_{1 b}+\bar{\rho}_{1 b} \bar{u}_{1 b}^{2}\right)^{2}+4 \bar{\rho}_{1 b} \bar{u}_{1 b}^{2}\left\{\frac{1}{2}\left(1-H^{-1} \bar{g}\right)-c_{p 0}\right\} \\
& \times\left\{c_{p 0} \frac{\bar{p}_{1 b}}{\left(1-H^{-1} g^{*}\right)}+\frac{1}{2} \bar{u}_{1 b}^{2} \bar{\rho}_{1 b}+\bar{\rho}_{1 b}\left(\bar{L} \bar{g}-\bar{L}_{1 b} g^{*}\right)\right\}\left(1-H^{-1} \bar{g}\right)
\end{aligned}
$$

and

$$
\begin{gathered}
\bar{\rho}=\frac{\bar{\rho}_{1 b} \bar{u}_{1 b}}{\overline{\bar{u}}\left(\bar{g}, g^{*}\right)}=\bar{\rho}\left(\bar{g}, g^{*}\right), \\
\bar{p}=\bar{p}_{1 b}+\bar{\rho}_{1 b} \bar{u}_{1 b}^{2}-\bar{\rho}_{1 b} \bar{u}_{1 b} \bar{u}\left(\bar{g}, g^{*}\right)=\bar{p}\left(\bar{g}, g^{*}\right), \\
\bar{T}=\frac{\bar{p}}{\bar{\rho}\left(1-H^{-1} \bar{g}\right)}=\bar{T}\left(\bar{g}, g^{*}\right) .
\end{gathered}
$$

On the other hand, the simplified rate equation in the droplet growth zone downstream of the shock preserves the form

$$
\begin{gathered}
\bar{g}=\bar{R}^{3}, \\
\partial \bar{R} / \partial \chi=\bar{\Omega}(\bar{p}, \bar{T}, \bar{g}),
\end{gathered}
$$

where $\bar{R}$ is the normalized radius of droplets defined in [10] together with $\bar{\Omega}(\bar{p}, \bar{T}, \bar{g})$. Substituting from Eqs. (72) and (73) into Eq. (75) and utilizing Eq. (74) yield

$$
\partial \bar{R} / \partial \chi=\bar{\Omega}\left[\bar{p}\left(\bar{g}, g^{*}\right), \bar{T}\left(\bar{g}, g^{*}\right), \bar{g}\right] \equiv \Omega^{* *}\left(\bar{g}, g^{*}\right) \equiv \Omega^{* * *}\left(\bar{R}, g^{*}\right)
$$

Integrating Eq. (76) from the shock front $(\chi=0)$ to a point with deformed coordinate $\chi$ downstream of the shock along the streamtube, we obtain

$$
\int_{\left(g^{*}\right)^{1 / 3}}^{(\bar{g})^{1 / 3}} \frac{d \sigma}{\Omega^{* * *}\left(\sigma, g^{*}\right)}=\chi
$$

Equation (77) gives the implicit solution of the rate equation in the form

$$
\bar{g}=\bar{g}\left(\chi, g^{*}\right)
$$


along the streamtube downstream of the shock. The values of the flow variables $\bar{u}$, $\bar{p}, \bar{\rho}$, and $\bar{T}$ at $\chi$ along a streamtube downstream of the shock can be calculated by direct substitution from Eq. (78) into Eqs. (69)-(73) provided that $g^{*}$ is known.

With the above given droplet growth shock structure solution along a typical streamtube downstream of the shock where $g^{*}$ is left to be determined, the construction of the network of streamtubes downstream of the shock in principle follows the same procedure of Sec. III. However, the calculations with forward and central difference formulas for various normal derivatives cannot be performed since such calculations occasionally involve crossing of the shock front, which is not allowed. Instead the calculations with normal derivatives should be performed by replacing the forward and central difference formulas upstream of the shock with backward difference formulas downstream of the shock. Thus the solution of the droplet growth zone downstream of the embedded, frozen, gas dynamic, oblique shock wave together with the formalism and method of solution of Sec. III modified by replacing the forward and central difference formulas for normal derivatives with the backward difference formulas completely determines the flow field and construction of the network of streamtubes downstream of the shock if $g^{*}$ is known. To be able to determine the correct value of $g^{*}$ along each streamtube, we discuss a shock fitting technique.

E. Shock fitting. The construction of the network of streamtubes downstream of the shock, as has been already pointed out, requires the value of the condensate mass fraction $g^{*}$ at the shock front along each streamtube. In determining $g^{*}$ we use a shock fitting technique originally introduced by Barschdorff [11] for iterative numerical calculations and analytically extended by the present authors [12] in the study of one-dimensional nozzle flows with nonequilibrium condensation.

We let $\chi_{s 2}$ be some point in the droplet growth zone downstream of the shock on a streamtube, and we denote the Mach number, based on a typical speed of sound defined by $\sqrt{\gamma \bar{T}}$, and the condensate mass fraction at this location by $\bar{M}_{s 2}$ and $\bar{g}_{s 2}$, respectively. The Barschdorff conditions for shock fitting of one-dimensional nozzle flow discussed in detail in [11] and [12] should be satisfied at $\chi_{s 2}$ on each streamtube that passes through the shock. These conditions can be conveniently written for the present work as

$$
\bar{M}_{s 2}\left(\bar{g}_{s 2}, g^{*}\right) \equiv \frac{\bar{u}^{2}\left(\bar{g}_{s 2}, g^{*}\right)}{\gamma \bar{T}\left(\bar{g}_{s 2}, g^{*}\right)}=\frac{\left(1-H^{-1} \bar{g}_{s 2}\right)}{\left[1+H^{-1} \bar{g}_{s 2}(\gamma-1)\right]}
$$

and

$$
\begin{aligned}
\left(\frac{1}{A} \frac{\partial A}{\partial \chi}\right)_{s 2}= & \left\{\frac{\tilde{Q}}{2\left(1+\tilde{Q} \bar{g}_{s 2}\right)} \cdot \frac{\left[1+\gamma+H^{-1} \bar{g}_{s 2}(\gamma-1)\right]}{\left[1+H^{-1} \bar{g}_{s 2}(\gamma-1)\right]}-\frac{H^{-1}}{\left(1-H^{-1} \bar{g}_{s 2}\right)}\right\} \\
& \times 3\left(\bar{g}_{s 2}\right)^{2 / 3} \Omega^{* * *}\left(\bar{g}_{s 2}^{1 / 3}, g^{*}\right),
\end{aligned}
$$

where

$$
\tilde{Q} \equiv \frac{\bar{L}_{1 b}}{c_{p 0}+\frac{1}{2} u_{c}^{2}} .
$$

In deriving conditions (79) and (80), $\bar{L}$ is treated as a constant. 
Further simplification of Eq. (80) is possible if we consider the expansion (63) of the normal area written as a function of the deformed coordinate $\chi$ as

$$
A(\chi)=\left(A_{1 b}\right)_{n}+(\partial A / \partial s)_{1 b} l^{-1} K^{1 / 3} \chi .
$$

The derivative $(\partial A / \partial s)_{1 b}$ can be evaluated from the geometry of Fig. 4 utilizing Eqs. (54) and (56).

$$
\left(\frac{\partial A}{\partial s}\right)_{1 b}=\frac{\left(A_{3}\right)_{n}-\left(A_{1 b}\right)_{n}}{\Delta s}=\frac{\left(\theta_{0}+\varepsilon-\theta_{1 a}\right)}{\Delta n_{c}\left(A_{0}\right)_{n}}\left(A_{1 b}\right)_{n} .
$$

Substituting from Eq. $(82)$ for $A\left(\chi_{s 2}\right)$ and its derivative $(\partial A / \partial \chi)_{s 2}$ together with Eq. (83) into Eq. (80), solving for $\chi_{s 2}$, and utilizing Eq. (77) at $\chi_{s 2}$, we obtain

$$
\begin{aligned}
\chi_{s 2}= & {\left[\frac{1}{3}\left(\bar{g}_{s 2}\right)^{-2 / 3}\left[\Omega^{* * *}\left(\bar{g}_{s 2}^{1 / 3}, g^{*}\right)\right]^{-1} \times\left\{\frac{\tilde{Q}}{2\left(1+\tilde{Q} \bar{g}_{s 2}\right)} \cdot \frac{\left[1+\gamma+H^{-1} \bar{g}_{s 2}(\gamma-1)\right]}{\left[1+H^{-1} \bar{g}_{s 2}(\gamma-1)\right]}\right.\right.} \\
& \left.\left.-\frac{H^{-1}}{\left(1-H^{-1} \bar{g}_{s 2}\right)}\right\}^{-1}-\frac{\Delta n_{c}\left(A_{0}\right)_{n}}{\left(\theta_{0}+\varepsilon-\theta_{1 a}\right)} l K^{-1 / 3}\right] \\
& =\int_{\left(g^{*}\right)^{1 / 3}}^{\left(\bar{g}_{s 2}\right)^{1 / 3}} \frac{d \sigma}{\Omega^{* * *}\left(\sigma, g^{*}\right)} .
\end{aligned}
$$

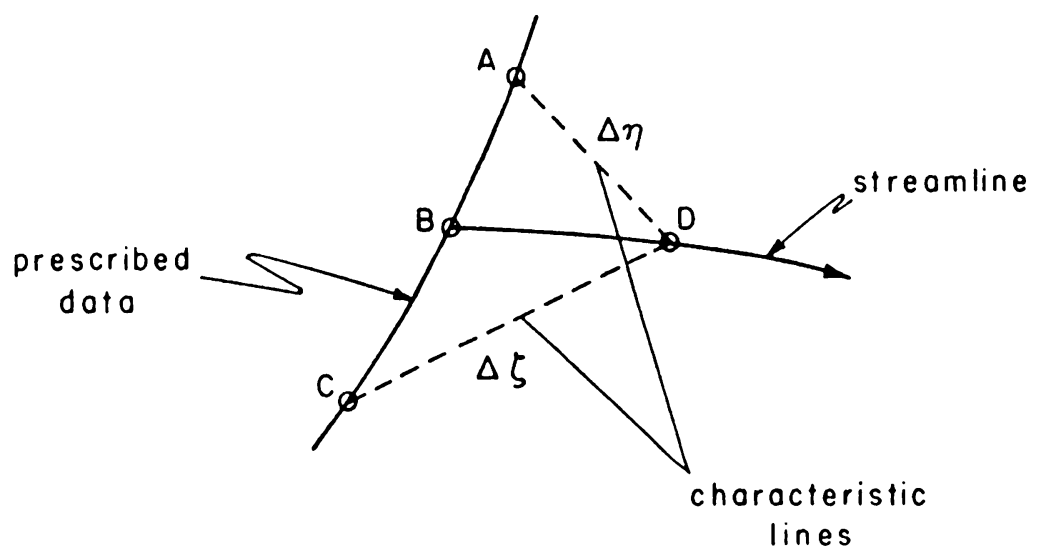

FIG. 5. Construction for finite difference calculation by the numerical method of characteristics.

Equations (79) and (84) provide two coupled equations to be solved for $\bar{g}_{s 2}$ and $g^{*}$ as functions of $\left(A_{0}\right)_{n}, \theta_{0}$, and the flow properties at 1a. The exact location of point 1 on the streamtube where the shock should be fitted is determined when the local value of the condensate mass fraction at this location agrees within a degree of accuracy with the value $g^{*}$ to be obtained by solving Eqs. (79) and (84) at the same location.

V. Qualitative comparison with the numerical method of characteristics. As we have already mentioned in the Introduction section, the numerical method of characteristics is a widely used method in calculating two-dimensional nonequilibrium supersonic flows. The detailed development of the method for nonequilibrium 
flows with only one relaxation rate equation can be found in Vincenti and Kruger [17] and for nonequilibrium flows with condensation is worked out in [16]. As shown in [16], the compatibility equations for the flow variables $u, \rho, p, g$, and $\theta$ can be conveniently written as

$$
\begin{gathered}
\rho u d u+d p=0, \\
u d g-\omega d s=0, \\
h+\frac{1}{2} u^{2}=\frac{c_{p 0} p}{\rho\left(1-H^{-1} g\right)}-L g+\frac{1}{2} u^{2}=\text { constant }
\end{gathered}
$$

on streamlines and

$$
\left[\frac{u^{2}}{a_{f}^{2}}-1\right]^{1 / 2} d p \pm \rho u^{2} d \theta-\frac{h_{g}}{h_{\rho}} a_{f} \omega\left(\begin{array}{l}
d \eta \\
d \zeta
\end{array}\right)=0
$$

on the characteristic lines,

$$
\frac{d n}{d s}=\mp \frac{1}{\left[u^{2} / a_{f}^{2}-1\right]^{1 / 2}},
$$

where $d \eta$ and $d \zeta$ are infinitesimal distances along the characteristics and

$$
\left.\begin{array}{rl}
h_{g} & \equiv \frac{c_{p 0} p H^{-1}}{\rho\left(1-H^{-1} g\right)^{2}}-L, \\
h_{\rho} & \equiv-\frac{c_{p 0} p}{\rho^{2}\left(1-H^{-1} g\right)}, \\
a_{f}^{2} & \equiv \frac{c_{p 0} p}{\rho\left(c_{p 0}+H^{-1} g-1\right)} .
\end{array}\right\}
$$

Written in finite difference form, Eqs. (85)-(88) provide five simultaneous equations for two-dimensional supersonic $\left(u>a_{f}\right)$ flows with nonequilibrium condensation in calculating $p, u, \theta, \rho$, and $g$ at a point $D$ when the data are known at points $A, B$, and $C$ on a noncharacteristic line (Fig. 5). The details for finite difference calculations can be found in Ferri [18] and Liepmann and Roshko [19].

The numerical method of characteristics discussed in [16] and above for nonequilibrium condensing flows is based on the assertion that the single nonequilibrium rate equation can be written in the form

$$
u \partial g / \partial s=\omega(p, \rho, g) \text {, }
$$

where $\omega$ is a known function. However, from consideration of the various fundamental concepts of production by nucleation and of subsequent droplet growth, such a rate equation cannot be written directly. Instead the condensate rate equation in this study is developed in integral form in the appendix where a power-law radius dependence of the droplet growth rate is included. For quasi-one-dimensional flows the area can be replaced by $A=u_{c} /(\rho u)$ from the continuity equation. Thus the integral condensation rate equation takes the form

$$
g(s)=\lambda^{3} u_{c} \int_{-\infty}^{s} \frac{\mathscr{M}(s, \xi)}{\rho(\xi) u(\xi)} \Sigma(\xi) \exp \left[-K^{-1} B(\xi)\right] d \xi
$$

on streamlines, where $\mathscr{M}(s, \xi)$ is given by Eq. (15). 
For numerical calculations with the method of characteristics, the integral condensation rate equation (92) is not convenient. Instead one seeks to reduce Eq. (92), if possible, into a system of differential equations on streamlines for finite difference calculations. It follows from Eqs. (92) and (15) that such a reduction is possible if $3 m$ is an integer, and in all other cases where the droplet growth rate depends on the radius, such a reduction seems not possible.

In the interval $1 / 2 \leq m \leq 1$, there are only two values of $m$ for which $3 m$ is an integer:

$$
\begin{aligned}
& m=1 \quad\left(\begin{array}{l}
\text { droplet growth rate is independent } \\
\text { of the droplet radius }
\end{array}\right) \\
& m=2 / 3 \quad\left(\begin{array}{l}
\text { droplet growth rate depends on } \\
\text { the radius as } r^{-1 / 2}
\end{array}\right) .
\end{aligned}
$$

In all other cases the equivalent system of differential equations for the integral condensation rate equation (92) seems impossible to obtain. In cases where the reduction is possible ( $m=1$ and $m=2 / 3$ ), the equivalent system of differential equations on streamlines, as worked out in detail in [16], can be easily obtained by applying Leibnitz's rule for differentiation under the integral sign to Eq. (92) successively.

From the results of [16] for $m=1$, it is well known (Oswatitsch [1] and Sislian and Glass [8]) that the rate equation (86) on streamlines can be replaced by a system of four differential equations for $g, C_{1}, C_{2}$, and $C_{3}$, namely,

$$
\begin{aligned}
d g & =3 \lambda^{3} u_{c} \Omega(s) C_{1}(s) d s, \\
d C_{1} & =2 \Omega(s) C_{2}(s) d s, \\
d C_{2} & =\Omega(s) C_{3}(s) d s, \\
d C_{3} & =\frac{\Sigma(s)}{\rho(s) u(s)} \exp \left[-K^{-1} B(s)\right] d s
\end{aligned}
$$

on streamlines. The initial conditions for $C_{1}, C_{2}$, and $C_{3}$ can be taken to be zero at the saturation point $s=s_{c}$. The function $\omega$ in this case $(m=1)$ is simply

$$
\omega \equiv 3 \lambda^{3} u_{c} \Omega(s) u(s) C_{1}(s) \text {. }
$$

Similarly when $m=2 / 3$, we find that the rate equation (86) on streamlines can be replaced by a system of three differential equations for $g, D_{1}$, and $D_{2}$ on streamlines, namely,

$$
\begin{aligned}
d g & =2 \lambda^{3} u_{c} \Omega(s) D_{1}(s) d s, \\
d D_{1} & =\Omega(s) D_{2}(s) d s, \\
d D_{2} & =\frac{\Sigma(s)}{\rho(s) u(s)} \exp \left[-K^{-1} B(s)\right] d s
\end{aligned}
$$

on streamlines. The initial conditions for $D_{1}$ and $D_{2}$ again can be taken to be zero at the saturation point $s=s_{c}$. The function $\omega$ in this case $(m=2 / 3)$ can be identified as

$$
\omega \equiv 2 u_{c} \lambda^{3} \Omega(s) u(s) D_{1}(s) .
$$


The numerical method of characteristics is very inconvenient for all cases different from the above discussed.

The proposed streamtube method, on the other hand, can be applied for all $m$ in the interval $1 / 2 \leq m \leq 1$ incorporating the radius dependence of the droplet growth law by a straightforward extension of the analysis of [10] for all $m$. Although the onset of condensation in two-dimensional nonequilibrium flows can be predicted by the numerical method of characteristics (Sislian and Glass [8]), the information to be gained from the solution by the proposed method will also contain the analytic structure of the asymptotic method of [10] so that measurements obtained over physically distinct regions could be compared with the asymptotic structure of the flow along each streamtube. In particular, Barschdorff's [11] one-dimensional shock fitting technique is easily incorporated to locate the oblique shock wave arising from compressive heat release in supercritical flows by the present method.

VI. Conclusions. The streamtube method for supersonic expansion flows on walls with nonequilibrium condensation is developed. The flow equations along each streamtube take the precise form of those for one-dimensional nozzle flows with nonequilibrium condensation for arbitrarily small cross-sectional area. The asymptotic solution of [10] for such flows with a two-dimensional consideration determines the flow field everywhere if the network of streamtubes is constructed simultaneously. In cases of relatively high initial humidity where compressive heat addition from condensation results in oblique shock waves (e.g., see Pouring [20]), the formalism and method of solution of the proposed method are supplemented by the derived oblique shock relations, and a shock fitting technique, originally introduced by Barschdorff [11], is used to determine the location of the oblique shock wave. A qualitative comparison with the widely used numerical method of characteristics suggests that the present method may have some advantages in incorporating the radius dependence of the droplet growth law, in exhibiting the analytic nature of the physically distinct zones of condensation from the asymptotic solution of [10], and in locating the position of the oblique shock wave.

The method proposed in this paper needs to be substantiated by actual numerical calculations. Unfortunately such numerical calculations are not available yet; however, it is expected that the computer experiment of the formalism and method of solution of this investigation will show quantitative agreement with the available data. Furthermore, the information to be obtained from such an experience may help in improving the experimental research and design procedures providing a satisfactory description of expansion flows on walls with nonequilibrium condensation.

Appendix. Integral condensate rate equation in normalized variables. The integral condensate rate equation (13) written in the form

$$
g^{\prime}\left(s^{\prime}\right)=\frac{1}{\Delta m^{\prime}} \int_{-\infty}^{s^{\prime}} d \xi^{\prime} m_{r}^{\prime}\left(s^{\prime}, \xi^{\prime}\right) 1 \cdot \Delta n^{\prime}\left(\xi^{\prime}\right) J^{\prime}\left(\xi^{\prime}\right)
$$

requires information on the rate at which clusters of critical size are nucleated and on the rate at which droplet growth ensues from these clusters. 
Various theories for the nucleation rate $J^{\prime}$ have the common functional form

$$
J^{\prime}=S^{\prime} J(p, T, g ; K)=S^{\prime} \Sigma(p, T, g) \exp \left[-K^{-1} B(p, T, g)\right],
$$

where $B(p, T, g)$ is the activation function, $K$ is the "nucleation parameter" assumed to be small, $S^{\prime}$ is a normalization constant, and $\Sigma(p, T, g)$ is a function of the independent thermodynamic coordinates normalized such that at the saturation point $\Sigma(1,1,0)=1$. In this investigation no specific choice is made regarding the preexponential factor $S^{\prime} \Sigma(p, T, g)$ and the activation function $B(p, T, g)$ since they vary considerably in different theories (e.g., see Volmer and Weber [21], Lothe and Pound [22], etc.).

Moreover, the mass of a droplet $m_{r}^{\prime}\left(s^{\prime}, \xi^{\prime}\right)$ at current point $s^{\prime}$ that originated at prior point $\xi^{\prime}$ along a streamtube for spherical droplets is by definition

$$
m_{r}^{\prime}\left(s^{\prime}, \xi^{\prime}\right)=\frac{4}{3} \pi \rho_{l}^{\prime}\left[r^{\prime}\left(s^{\prime}, \xi^{\prime}\right)\right]^{3},
$$

where $r^{\prime}\left(s^{\prime}, \xi^{\prime}\right)$ is the current radius of a droplet at $s^{\prime}$ that originated at $\xi^{\prime}$ and $\rho_{l}^{\prime}$ is the true mass density of the nondispersed condensed phase. The droplet growth law which determines the radius $r^{\prime}\left(s^{\prime}, \xi^{\prime}\right)$ is obtained from the mass and energy balance of the droplet. A detailed discussion of the balance laws can be found in Hill [23]. For steady flow the droplet growth rate equation obtained from the mass balance of the droplet can be written in functional form as

$$
\partial r^{\prime} / \partial s^{\prime}=F_{1}\left(p^{\prime}, T^{\prime}, g^{\prime}, T_{D}^{\prime}, r^{\prime}\right),
$$

where $T_{D}^{\prime}$ is the actual temperature of the droplet. Moreover the widely accepted quasi-equilibrium assumption for energy balance yields

$$
T_{D}^{\prime}=T_{D}^{\prime}\left(p^{\prime}, T^{\prime}, g^{\prime}, r^{\prime}\right) \text {. }
$$

Substituting from Eq. (A5) into Eq. (A4), we obtain

$$
\partial r^{\prime} / \partial s^{\prime}=G_{1}\left(p^{\prime}, T^{\prime}, g^{\prime}, r^{\prime}\right)
$$

where

$$
G_{1}\left(p^{\prime}, T^{\prime}, g^{\prime}, r^{\prime}\right) \equiv F_{!}\left[p^{\prime}, T^{\prime}, g^{\prime}, T_{D}^{\prime}\left(p^{\prime}, T^{\prime}, g^{\prime}, r^{\prime}\right), r^{\prime}\right] .
$$

Equation (A6) exhibits radius dependence on the droplet growth rate as well as the dependence on the independent thermodynamic coordinates $p^{\prime}, T^{\prime}$, and $g^{\prime}$. The radius dependence on the droplet growth rate given by Eq. (A6) can be exploited from the excellent summary by Gyarmathy [3]. In this summary it is shown that the radius dependence on the droplet growth rate is governed by the Knudsen number Kn defined by

$$
\mathrm{Kn} \equiv l_{m}^{\prime} / 2 r^{\prime},
$$

where $l_{m}^{\prime}$ is the mean free path of the mixed vapor and gas molecules. The discussion in Gyarmathy [3] shows

$$
\text { when } \mathrm{Kn} \ll 1, \quad \partial r^{\prime} / \partial s^{\prime} \propto 1 / r^{\prime}
$$

and 
For convenience, we generalize these results to include all ranges of the Knudsen number by simply assuming a power law radius dependence on the droplet growth rate and write Eq. (A6) in the form

$$
\partial r^{\prime} / \partial s^{\prime}=\left(r^{\prime}\right)^{-n} G\left(p^{\prime}, T^{\prime}, g^{\prime}\right)
$$

where $0 \leq n \leq 1$. In particular $n=0$ corresponds to the case $\mathrm{Kn} \gg 1$ and $n=1$ to the case $\mathrm{Kn} \ll 1$.

We now carry out the normalization $r \equiv r^{\prime} / r_{d}^{\prime}$,

$$
\begin{gathered}
\Omega(s) \equiv \Omega[p(s), T(s), g(s)] \equiv \frac{G\left(p_{c}^{\prime} p, T_{c}^{\prime} T,\left(\mu_{v} /\left(\mu_{0} H\right)\right) g\right)}{G\left(p_{c}^{\prime}, T_{c}^{\prime}, 0\right)}, \\
\lambda_{1} \equiv \frac{s_{0}^{\prime}}{r_{d}^{(n+1)}} G\left(p_{c}^{\prime}, T_{c}^{\prime}, 0\right),
\end{gathered}
$$

where $r_{d}^{\prime}$ is determined from

$$
\frac{(4 / 3) \pi \rho_{l}^{\prime} r_{d}^{\prime 3} S^{\prime} s_{0}^{\prime} \Delta n_{c}^{\prime}}{\Delta m^{\prime}}=\frac{\mu_{v}}{\mu_{0} H} \equiv \frac{g^{\prime}}{g}
$$

With the above normalization the droplet growth rate equation can be written as

$$
\partial r / \partial s=\lambda_{1} r^{-n} \Omega(p, T, g), \quad 0 \leq n \leq 1,
$$

where $\lambda_{1}$ is the "droplet growth parameter" $\equiv($ flow time/droplet growth time) assumed to be large. Integrating Eq. (A8) along a streamtube and neglecting the initial critical radius at $\xi$, we obtain

$$
r(s, \xi)=\lambda\left[\int_{\xi}^{s} \Omega(\eta) d \eta\right]^{m}, \quad \frac{1}{2} \leq m \leq 1,
$$

where

$$
\lambda \equiv\left[\lambda_{1}(1+n)\right]^{1 /(n+1)} \quad \text { and } \quad m \equiv \frac{1}{(n+1)} .
$$

Substitution from Eq. (A9) into Eq. (A3) with the above normalization yields

$$
m_{r}^{\prime}\left(s^{\prime}, \xi^{\prime}\right)=\frac{4}{3} \pi \rho_{l}^{\prime} r_{d}^{\prime 3} \lambda^{3}\left[\int_{\xi}^{s} \Omega(\eta) d \eta\right]^{3 m}, \quad \frac{1}{2} \leq m \leq 1 .
$$

Now if Eq. (A10) for $m_{r}^{\prime}\left(s^{\prime}, \xi^{\prime}\right)$ and Eq. (A2) for $J^{\prime}\left(\xi^{\prime}\right)$ together with the above normalization are substituted into Eq. (A1), we obtain the integral condensate rate equation in normalized form as

$$
g(s)=\lambda^{3} \int_{-\infty}^{s} \mathscr{M}(s, \xi) \Sigma(\xi) A(\xi) \exp \left[-K^{-1} B(\xi)\right] d \xi,
$$

where

$$
\mathscr{M}(s, \xi)=\left[\int_{\xi}^{s} \Omega(\eta) d \eta\right]^{3 m}, \quad \frac{1}{2} \leq m \leq 1 .
$$

The integral condensate rate equation (A11) incorporates the radius dependence on the droplet growth rate in the form of a power law. The radius dependence is generally left out entirely in the literature. In particular, $m=1$ corresponds to droplet growth rates in flows where $\mathrm{Kn} \gg 1$ and $m=1 / 2$ to droplet growth rates in flows where $\mathrm{Kn} \ll 1$. 


\section{REFERENCES}

[1] K. Oswatitsch, Z. Angew. Math. Mech. 22, 1 (1942)

[2] P. P. Wegener, Nonequilibrium flows, Vol. 1, Part 1, Marcel Dekker, New York and London, 1969

[3] G. Gyarmathy, in M. J. Moore and C. H. Sieverding (Eds.), Two-phase steam flow in turbines and separators, Hemisphere Pub. Corp., Washington and London, 1976

[4] F. Bartlmä, Proc. 11th Internat. Congr. Appl. Mech., Munich, 1964

[5] A. V. Kurshakov, G. A. Saltanov, and R. A. Tkalenko, Prikl. Mekh. Tekh. Phys. 5, 117 (1971)

[6] L. T. Smith, AIAA J. 9, 2035 (1971)

[7] L. M. Davydov, Fluid Mech., Soviet Res., Vol. 1, No. 1 (1972)

[8] J. P. Sislian and I. I. Glass, AIAA J. 14, 1731 (1976)

[9] P. A. Blythe and C. J. Shih, J. Fluid Mech. 76, 593 (1976)

[10] J. H. Clarke and C. F. Delale, Phys. Fluids 29 (5), 1398 (1986)

[11] D. Barschdorff, Forsch. Ingenieur Wes. 37, 146 (1971)

[12] J. H. Clarke and C. F. Delale, Phys. Fluids 29, 1414 (1986)

[13] D. Greenspan, Discrete numerical methods in physics and engineering, Academic Press, New York, 1974

[14] G. D. Smith, Numerical solution of partial differential equations, Oxford University Press, London, 1978

[15] P. D. Lax, Weak solutions of nonlinear hyperbolic equations and their numerical computation, Comm. Pure Appl. Math. 7, 159-193 (1954)

[16] C. F. Delale, Ph.D. Thesis, Brown University (1983)

[17] W. G. Vincenti and C. H. Kruger, Jr., Introduction to physical gas dynamics, Wiley and Sons, New York, London and Sydney, 1965

[18] A. Ferri, Elements of aerodynamics for supersonic flows, Macmillan, New York, 1949

[19] H. W. Liepmann and A. Roshko, Elements of gas dynamics, Wiley and Sons, New York, London, and Sydney, 1957

[20] A. A. Pouring, Phys. Fluids 8, 1802 (1965)

[21] M. Volmer and A. Weber, Z. Physik Chem. A119, 227 (1926)

[22] J. Lothe and G. M. Pound, in A. C. Zettlemoyer (Ed.), Nucleation, Marcel Dekker, New York and London, 1969

[23] P. G. Hill, J. Fluid Mech. 25, 593 (1966) 